MODELING, IDENTIFICATION AND CONTROL, 2003, vOL. 24, NO. 1, 15-26

doi:10.4173/mic.2003.1.2

\title{
The Ski Base Structure Analyser (SSA)†
}

\author{
DAG ANDERS MOLDESTAD and SVEINUNG LØSET
}

Keywords: Image processing, tribology, roughness, ski, interferometry

\begin{abstract}
A Ski base Structure Analyser (SSA) utilising laser technology has been developed to analyse the detailed structure of a stone-ground ski base. The SSA displays the measured surface as an image with $739 \times 570$ pixels, where each pixel can have a grey level value from 0 to 255 . The value of each pixel is proportional to the height at the corresponding point of the measured surface. Various roughness parameters can be calculated from the measured surface image in order to characterise the surface topography. Some of these parameters are described in this paper. Further the paper contains examples of calculated SSA parameters from a typically measured surface image using MATLAB.
\end{abstract}

\section{Introduction}

For skis applied in competitions the requirements to the sliding surfaces are high. One of the major ski parameters is the ski base structure. The ski base structure contains grooves aligned along the ski that is in the order of $10^{-1} \mathrm{~mm}$ wide and $10^{-2}-10^{-1} \mathrm{~mm}$ deep. It is important to be able to characterise the surface topography and roughness of this structure, for instance in order to select competition skis with optimum sliding properties under different snow and weather conditions.

Systematic measurements of high-quality ski base structures have been paid very little attention to in the literature. However, Mathia et al. (1989 and 1992) reported the development of a 3-D profilometer for systematic study of micro and macro topography of ski bases, but they did not characterise different types of ski base structures or study the effect of different ski base structures on ski base sliding friction in their papers. Surface topography has important implications for wear, friction, lubrication, fatigue, sealing, painting and bearing surfaces, and related measurement problems exist in metals, plastics as well as electronics industry.

Both non-contact and contact methods are used for measuring the surface structure of an object. The contact method is based on a sensor head that traverses the surface while in contact. Normally the sensor head or stylus has a tip radius of about $2 \mu \mathrm{m}$ and a static load of less than $0.0007 \mathrm{~N}$ (Hamrock, 1994). Such a sensor head would not give a correct measurement result if the object material is very soft, or the movement of the sensor head is obstructed during the measurement. This may be a problem for ski bases of polyethylene prepared with glider products based on fluor or hydrocarbons. Non-contact measurement methods are therefore preferable in that case.

It is well known to use phase-measurement interference (PMI) techniques to

*Department of Civil and Transport Engineering, Norwegian University of Science and Technology, Trondheim, Norway.

$\dagger$ An early version of this article was presented at Nordic Matlab Conference 2001, October 2001, Oslo, Norway. 
detect surface characteristics, see e.g. Creath (1988). However, no appropriate portable method/instrumentation has so far existed for performing rapid, repeatable and nondestructive measurements of 3-D surface textures of sliding surfaces, i.e. skis, snow boards or similar equipment made for sliding on snow.

Mercer and Beheim (1992) described a system for surface topography measurement based on phase shifting, projected fringes and use of fibre optics. A projected fringe interferometer designed for measuring the topography of an object shifts stepwise and periodically the phase angle $\theta$ between two light beams emitted from the same source. The steps are $\pi / 2$ radians $\left(90^{\circ}\right)$ apart, and at each step a video image of the fringes is registered and stored. Photo detectors measure either the phase angle $\theta$ of the beams or $2 \theta$, and one of these angles is used to control one of the light beams so that the $90^{\circ}-\theta$ angle is accurately maintained. A camera, computer, phase modulator and phase controller establish closed-loop control of $\theta$. A calibration reference is found by measuring the phase matrix of a plane surface.

The phase controller described by Mercer and Beheim gives a precise phase shift, but do require specially designed electronics (Figure 2 in Mercer and Beheim, 1992) that hampers a flexible integration with present portable computers. A D/A card and a framegrabber card can easily control our measurement system. Thereby a compact and portable system can be obtained, where:

- A piezoelectric transducer controls the phase shift between the object beams and the reference beams by adjusting the position of a mirror in the path of the reference beams.

- A D/A-card in a portable computer controls the piezoelectric transducer.

The present paper gives an introduction to the ski base structure analyser (SSA), and the majority of the paper is extracted from the dr. thesis of Moldestad (1999).

\section{Basic Theory of the SSA Instrumentation and Measurement Technology}

The purpose of the development of the SSA has been to provide a portable measurement device that can give rapid, precise, repeatable and non-destructive 3-D measurements of surface topography. Furthermore the device was intended to execute the measurements statically, i.e. without relative movement between the device and the object.

The SSA applies a method and instrumentation that is specifically designed for measuring texture and roughness of black ski base surfaces, but measurements can also be performed on other one-coloured, dim surfaces. By measuring several test fields on the ski base surface, a sufficient precise estimate of the roughness characteristics of the total ski base surface can be given. The instrumentation of the SSA is designed to be compact and easily portable in order to do measurements independent of special laboratory facilities. Furthermore the SSA has a physical design that allows different dimensions and flexible positioning of the sliding surfaces that shall be measured.

The SSA measurement provides both a graphical presentation (see Figure 1) and a numerical characterisation (surface roughness or texture) of the measured surface. Characterisation of ski base structure topography by the SSA is further described in Section 3. 


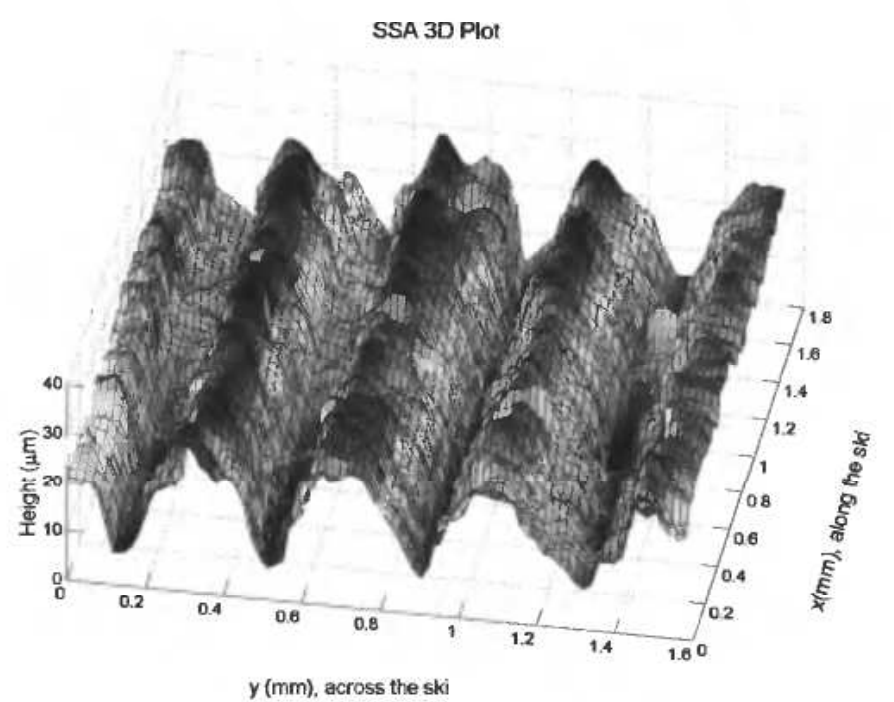

Figure 1. Graphical presentation of the SSA measurement result.

The SSA measurement technology is in the following described by referring to Figures 2 and 3, where:

- Figure 2 shows the major elements of the SSA technology and how it interacts with the measurement object.

- Figure 3 shows a block diagram of the optical unit (interferometer) that transforms the laser beam to projected fringes.
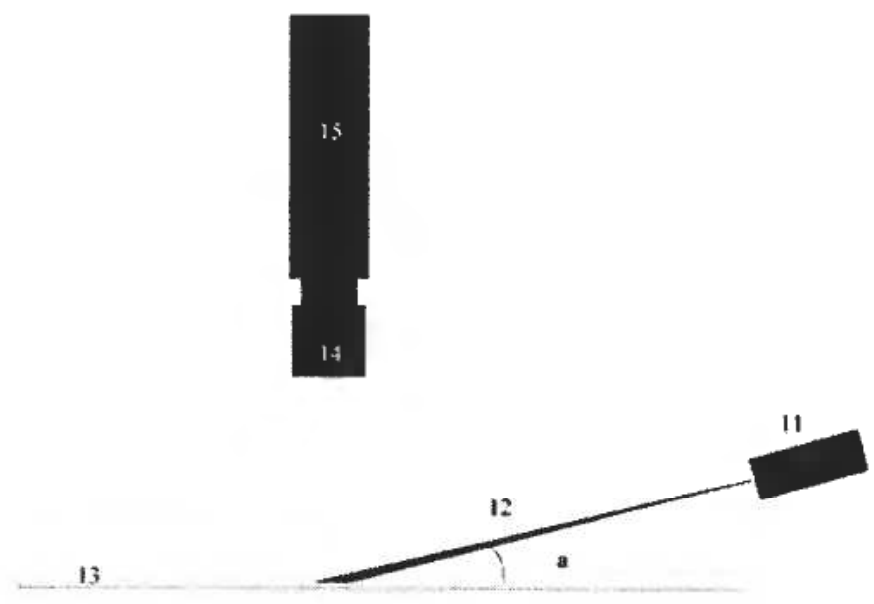

Figure 2. Major elements of the SSA technology.

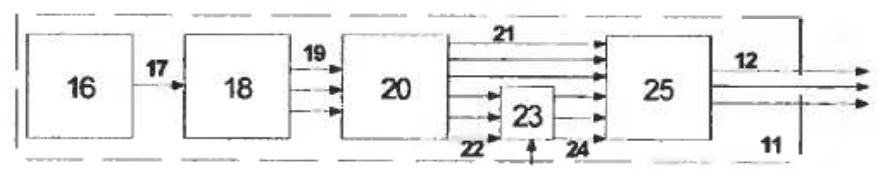

Figure 3. Possible flow chart for an optical unit (interferometer) that transforms a laser beam to projected fringes. 
In Figure 2 we see that the optical unit 11 project an area of the ski base 13 with an interference pattern in form of projected fringes. The optical unit 11 is designed as described in Figure 3. The projection 12, which is further described below, is emitted by the optical unit 11 at an angle $a$ to the surface 13. The structure of the measurement surface deflects the interference pattern in form of projected fringes, and the measurement surface with deflected pattern is registered at different phase shifts (see also the explanation of Figure 3) with the camera lens 14 of the CCD-camera 15. The CCDcamera 15 is connected to a framegrabber in a portable computer where further registration and processing of data are performed as described below.

The CCD-camera 15 with camera lens 14 registers images from a defined area of a surface 13 , e.g. an area of approximately $11 \times 15 \mathrm{~mm}$ or larger. Normally this will provide sufficient amount of data to characterise the surface area of interest accurately. A sliding surface may well have different roughness at different test areas on the surface, e.g. on the forebody and the afterbody of the ski. Thus measurements should be provided from different areas of the sliding surface in order to obtain optimum characteristics and knowledge of the sliding surface.

Figure 3 shows a possible flow chart for an optical unit 11 (interferometer) that transforms a laser beam to projected fringes. A laser 16 emits a beam 17 to a microscope objective 18 where the beam is scattered 19. The scattered beams enter the beam splitter 20 that splits the beams into two parts, object beams 21 and reference beams 22 . The two parts have the same intensity. The reference beams 22 are phase shifted in a systematic manner relative to the object beams 21 by a piezoelectric transducer 23 that is controlled by a D/A-card in a portable computer. The setup in the portable computer is not shown. The optical phase shift angle $\alpha$ can start between 0 and $90^{\circ}\left(\alpha_{1}\right)$ and then be increased in steps, e.g. by 90,180 and $270^{\circ}$.

The object beams 21 and the phase shifted reference beams 24 are then combined in a beam combiner 25 where they interfere after having propagated different distances. This causes an interference pattern in form of projected fringes 12 on the surface 13. The intensity of a pixel in the image registered by the CCD-camera (15), is given by:

$$
I_{1}(x, y)=I_{0}(x, y)+I_{a}(x, y) \cos \left(\alpha^{\prime}(x, y)\right)
$$

where:

$I_{1}(x, y)=$ intensity of an image pixel when the piezoelectric transducer has given the reference beams an optical phase shift, $0<\alpha=\alpha_{1}<90^{\circ}$, with respect to the object beams

$I_{0}(x, y)=$ intensity of an image pixel without projection from the optical unit 11

$I_{a}(x, y)=$ modulation amplitude in an image pixel for the fringe pattern that the optical unit 11 projects

$\alpha^{\prime}(x, y)=$ phase difference between the object beams 21 and the phase shifted reference beams 24 in an image pixel, radians or ${ }^{\circ}$

$(x, y)=$ position of an image pixel

The phase shift $\alpha$ can for instance be increased stepwise by $90^{\circ}$ in such a way that four images with different intensities $I_{1}(x, y), I_{2}(x, y), I_{3}(x, y)$ and $I_{4}(x, y)$ are registered with the camera (15). From these images a computer can calculate the phase difference $\alpha^{\prime}(x, y)$ between the object and phase shifted reference beams in each image pixel: 


$$
\alpha^{\prime}(x, y)=\arctan \left[\frac{I_{3}(x, y)-I_{1}(x, y)}{I_{2}(x, y)-I_{4}(x, y)}\right]
$$

This method for calculating the phase difference $\alpha^{\prime}(x, y)$ is called a 4-step technique (Creath, 1988). The phase difference can also be estimated by a 3-step technique. For more details, see e.g. Creath (1988).

Equation (2) gives the phase difference $\alpha^{\prime}(x, y)$ in the different image pixels values between $-\pi$ and $\pi$. For $\alpha^{\prime}(x, y)<0,2 \pi$ is added, thus $\alpha^{\prime}(x, y)$ is assigned values between 0 and $2 \pi$. The system then transforms the adjusted $\alpha^{\prime}(x, y)$ linearly to image pixel values between 0 and the maximum value of the grey scale, $g_{\max }$. The phase matrix of the object $O_{\alpha}(x, y)$ is then found as:

$$
O_{\alpha}(x, y)=\alpha^{\prime}(x, y)\left(\frac{g_{\max }}{2 \pi}\right)
$$

For a system with an 8-bit grey tone scale, $g_{\max }$ equals 255 .

The phase matrix of the object, $O_{\alpha}(x, y)$, is compared with the phase matrix of a plane reference surface, $R_{\alpha}(x, y)$, that is found with the same procedure as $O_{\alpha}(x, y)$. $R_{\alpha}(x, y)$ is stored in advance in the computer program that controls the measurement procedure. The program user can therefore choose between generating a new phase matrix for a plane reference surface or using the stored phase matrix from an earlier calibration of a plane reference surface. The height matrix, $z(x, y)$, is obtained in the following way:

$$
\begin{gathered}
\beta(x, y)=\left(\frac{2 \pi}{g_{\max }}\right)\left(O_{a}(x, y)-R_{a}(x, y)\right) \\
z(x, y)=\beta(x, y) \frac{d z}{2 \pi}
\end{gathered}
$$

where:

$$
\begin{aligned}
& \beta(x, y)=\text { phase difference caused by the texture of the measured object, radians } \\
& \text { or }^{\circ} \\
& d z \quad=\text { equidistance, e.g. } 45 \mu \mathrm{m}
\end{aligned}
$$

The equidistance $d z$ is found from a calibration procedure where the user moves an object manually in the $z$-direction with a micrometer system, while a new image is registered after each movement. When the image repeats itself after the projected fringes have moved one period in the image, the displacement in the $z$-direction can be read on the micrometer system, and the equidistance $d z$ can be found. This calibration procedure is performed initially for a new instrument and later say once a year. The value of $d z$ is stored in the computer program that controls the measuring device. In the prototype $d z$ is set to $45 \mu \mathrm{m}$. Surface topography of sliding surfaces will normally be in the range $0-45 \mu \mathrm{m}$.

$O_{\alpha}(x, y)$ and $R_{\alpha}(x, y)$ in equation (4a) is assumed to take on values between 0 and $g_{\max }$. Thus the quadrants must be considered in the subtraction of the phase matrices, i.e. for $\beta(x, y)<0$ in equation ( $4 \mathrm{a}), 2 \pi$ must be added.

In order to reconstruct the surface topography of the measured object correctly, $z(x, y)$ needs to be unwrapped. This is performed by correcting $\beta(x, y)$ when the 
phase difference between adjacent pixels is larger than $\pi$. More details can be found in e.g. Creath (1988). After $z(x, y)$ has been unwrapped, the surface topography of the sliding surface can be displayed in 3-D as e.g. shown in Figure 1. The ski base structure topography can also be characterised numerically. This is as mentioned earlier further described in Section 3.

\section{Basic Theory of the SSA Characterisation of Ski Base Structure Topography}

A number of different parameters can be used to characterise the ski base structure topography. Some of the most used parameters are described in this section. Most of the parameters are described for two dimensions, i.e. a row or a column of the height matrix $z(x, y)$ or a submatrix of $z(x, y)$. Parameters that are extended to three dimensions, i.e. the whole height matrix $z(x, y)$ or a submatrix of $z(x, y)$, are calculated according to the same principles as for two dimensions. All parameters are calculated using MATLAB. From the discrete set of surface height registrations of a surface profile across the ski $\left(z_{1}, z_{2}, \ldots, z_{N}\right)$, three different surface or structure parameters are commonly used:

Arithmetic mean roughness, $R_{a}$, defined by:

$$
R_{a}=\frac{1}{N} \sum_{i=1}^{N}\left|z_{i}\right|
$$

root mean square (rms) roughness, $R_{q}$ given by:

$$
R_{q}=\sqrt{\frac{1}{N} \sum_{i=1}^{N} z_{i}^{2}}
$$

and the maximum peak-to-valley height, denoted $R_{t}$ :

$$
R_{t}=\max (z)-\min (z)
$$

All discrete height registrations $\left(z_{1}, z_{2}, \ldots, z_{N}\right)$ of the surface profile refer to a reference line. The reference lines used by the SSA give a mean value of zero for $z_{i}$.

The directional orientation of the surface roughness can be found by computing the 2-D Fourier transform of the measured surface image:

$$
G(u, v)=\frac{1}{M N} \sum_{x=0}^{M-1} \sum_{y=0}^{N-1} g(x, y) \exp \left[-j 2 \pi\left(\frac{u x}{M}+\frac{v y}{N}\right)\right]
$$

where:

$g(x, y)=$ grey level value of a pixel in the measured surface image, i.e. the height matrix $z(x, y)$ from the SSA measurement transformed to an 8-bit image

$M \quad$ number of sampled points in the $x$-direction, i.e. number of columns in the measured surface image

$N \quad=$ number of sampled points in the $y$-direction, i.e. number of rows in the measured surface image

$u \quad=$ frequency variable in the $x$-direction, $u=0,1,2, \ldots, M-1$

$v=$ frequency variable in the $y$-direction, $v=0,1,2, \ldots, N-1$

$j=\sqrt{-1}$

and subsequently the spectrum of the 2-D Fourier transform:

$$
|G(u, v)|-\left|R^{2}(u, v)+I^{2}(u, v)\right|
$$




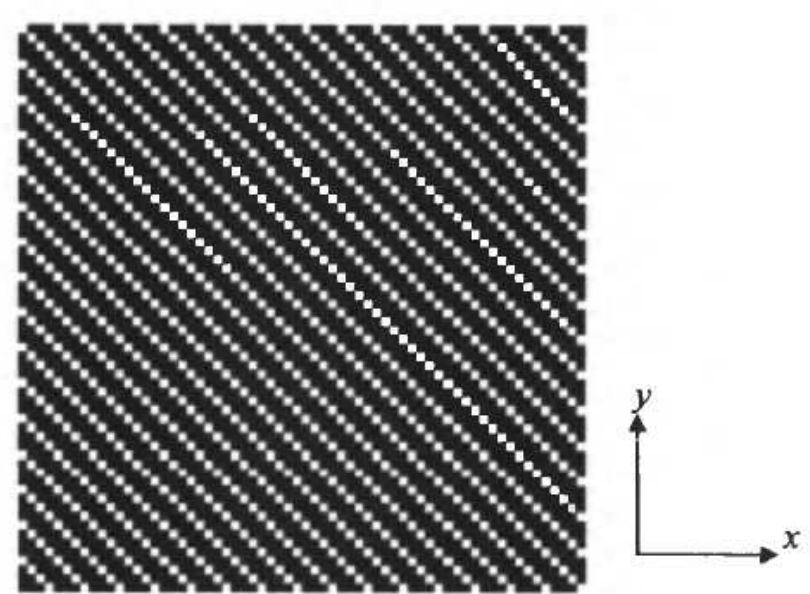

Figure 4. Imaginary surface image where heights at different surface points are described by grey tones in corresponding surface image pixels.

where:

$$
G(u, v)=R(u, v)+j I(u, v)
$$

Figure 4 shows an imaginary surface image where the heights at the different surface points are described by the grey tones in the corresponding surface image pixels. Light grey tones indicate high points, while dark grey tones indicate low points. The surface illustrated in Figure 4 has a texture with a characteristic direction of $-45^{\circ}$ relative to the $x$-axis and a characteristic period of $2 \sqrt{ } 2$ pixels perpendicular to the characteristic direction, if the resolution in the $x$ - and $y$-direction of the surface image is assumed equal. Figure 5 shows the spectrum of the 2-D Fourier transform for the surface image in Figure 4. We see that the surface image in Figure 4 gives rise to three points in the spectrum shown in Figure 5. The point in the middle of the

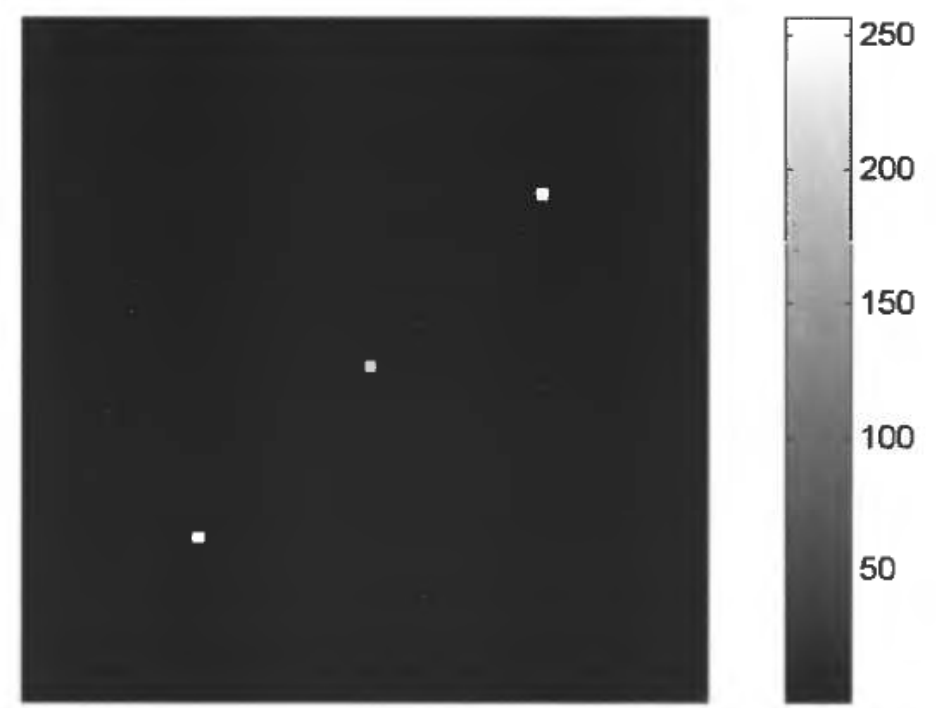

Figure 5. Spectrum of the 2-D Fourier transform of the imaginary surface image in Figure 4. 
spectrum, from now on entitled the origin, corresponds to the static component or the background of the measured surface. The two other points, that actually are one point due to the symmetry round the origin in a centred 2-D Fourier transform spectrum, can be used to find the characteristic direction and the characteristic period perpendicular to the characteristic direction for the surface.

A real surface can be interpreted as a collection of surfaces with different characteristic directions, characteristic periods perpendicular to characteristic directions and strengths. Bright points, i.e. points with high values, found in the 2-D Fourier spectrum of a measured surface image often indicate interesting properties distinctive for the measured surface. The characteristic direction of the bright point in the spectrum is related to the characteristic angle $\phi$ relative to the $x$-axis for the measured surface. The characteristic angle $\phi$ indicates the characteristic direction for the measured surface. If the bright point is located in the first (and third) quadrant relative to the origin in the spectrum, $\phi$ is given by:

$$
\phi=\delta-\frac{\pi}{2}
$$

where:

$\delta=$ angle relative to the $x$-axis for a vector from the origin to the bright point in the 2-D Fourier spectrum of a measured surface image, the angle must be adjusted accordingly if the resolution of the measured surface image is different in the $x$ - and $y$-direction, radians

If the bright point is located in the fourth (and second) quadrant relative to the origin in the spectrum, $\phi$ is given by:

$$
\phi=\frac{\pi}{2}-\delta
$$

The characteristic period perpendicular to the characteristic direction for the measured surface, $T(\mathrm{~mm})$, is given by:

$$
T=\frac{1}{\sqrt{\left(\frac{x_{c}}{N x_{r}}\right)^{2}+\left(\frac{y_{c}}{M y_{r}}\right)^{2}}}
$$

where:

$x_{r}=$ resolution in the $x$-direction, $\mathrm{mm} /$ pixel

$y_{r}=$ resolution in the $y$-direction, $\mathrm{mm} / \mathrm{pixel}$

$x_{c}=x$-coordinate relative to the origin for a bright point in the 2-D Fourier spectrum of a measured surface image, pixels

$y_{c}=y$-coordinate relative to the origin for a bright point in the 2-D Fourier spectrum of a measured surface image, pixels

\section{Example on Use}

A SSA-measured surface image has $739 \times 570$ pixels. Let us consider a part of such an image consisting of $128 \times 128$ pixels. Figure 6 shows a $3-D$ plot of this surface. Figure 7 displays the same surface as a grey level image, while the histogram of the surface is shown in Figure 8. The spectrum of the 2-D Fourier-transform of 


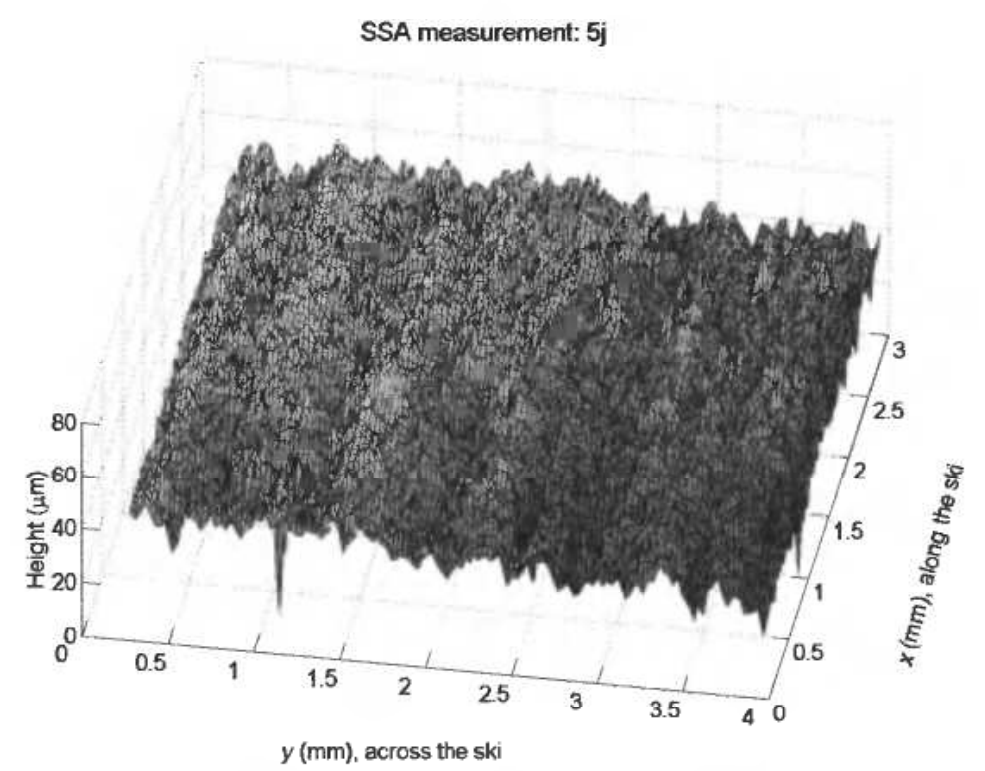

Figure 6. 3-D plot of a part of a SSA-measured surface image consisting of $128 \times 128$ pixels.

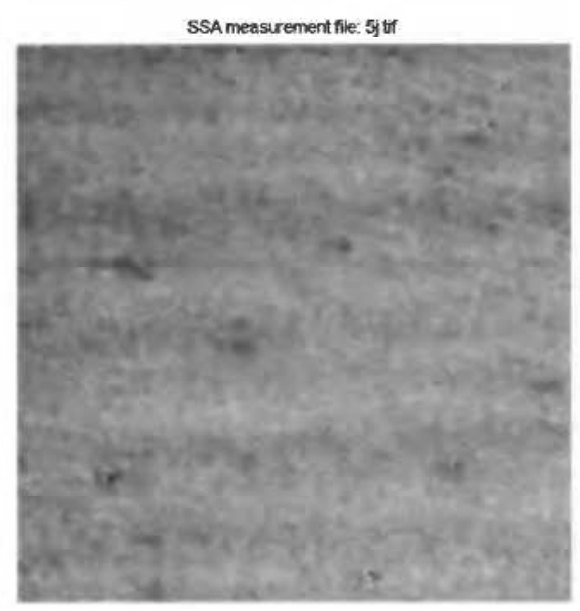

Figure 7. The surface in Figure 6 shown as a grey level image.

the surface is depicted in Figure 9. The bright point in the first (and third) quadrant of the spectrum in Figure 9 is used to find the characteristic angle $\phi$ and the characteristic period $T$ of the surface. These two parameters can be found in Table 1 together with the maximum strength of the bright point in the spectrum, $\log (|G(u, v)|)$, and different variants of the surface parameters $R_{q}, R_{a}$ and $R_{t}$. All the parameters have been calculated using MATLAB.

\section{Discussion}

The SSA example shows that the 2-D Fourier transform can be used to find the characteristic angle $\phi$ and the characteristic period $T$ of rcal ski base surfaces. $\bar{R}_{a, y}$ 


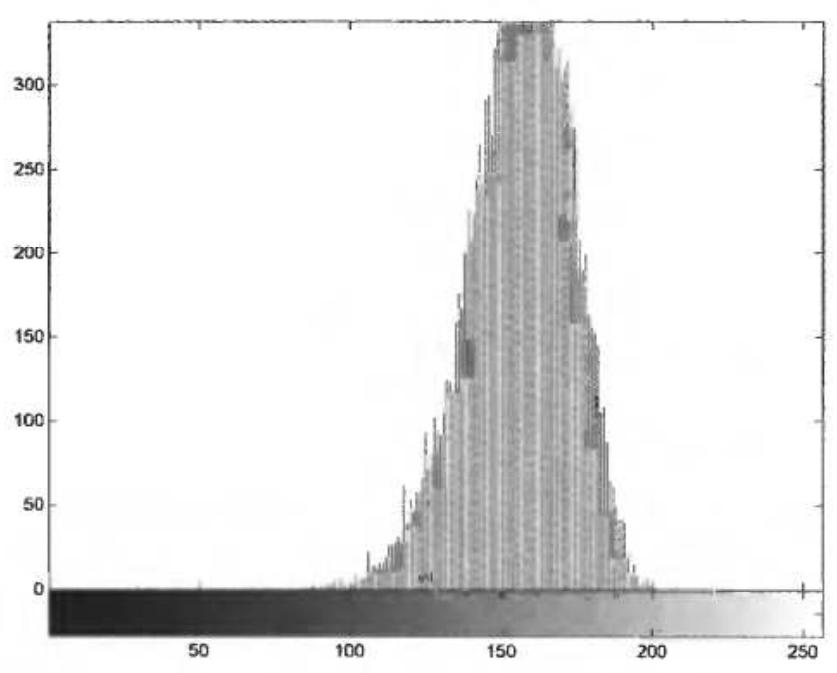

Figure. 8. The histogram of the surface in Figure 7. The grey tones are indicated on the $x$-axis, while the number of image pixels with different grey tones are given on the $y$-axis.

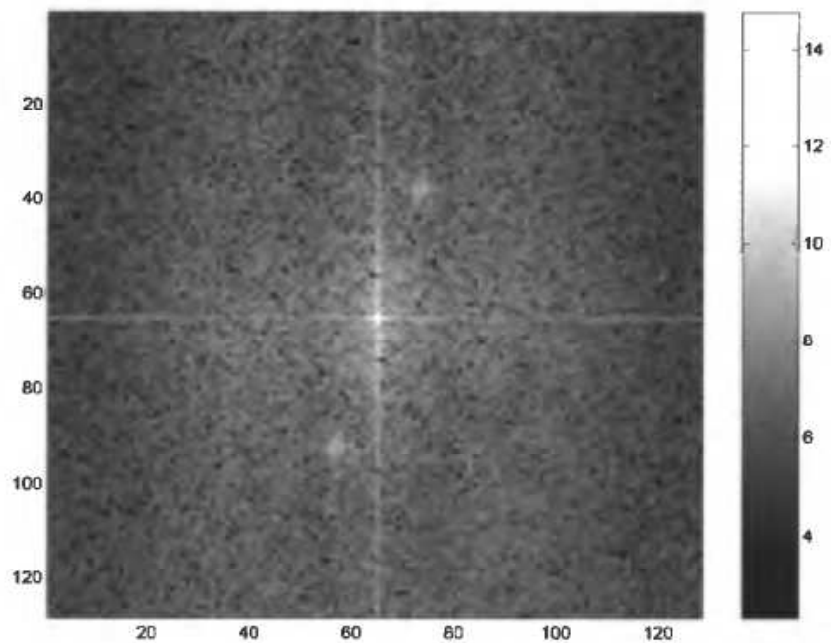

Figure 9. The spectrum of the 2-D Fourier transform of the surface in Figure 7.

and $\bar{R}_{a, y s}$ are the most commonly used parameters in simple analysis of ski base structure roughness. $\bar{R}_{a, y}$ is defined according to individual reference lines parallel to the $y$-axis, while $\bar{R}_{a, y s}$ is defined according to individual possibly sloping reference lines relative to the $y$-axis. Differences between the $\bar{R}_{a, y s^{-}}$and $\bar{R}_{a, y}$-values indicates that the ski base surface is not plane. Typically $\bar{R}_{a, y}$ equals $\bar{R}_{a, y s}$ for skis that are properly plane ground before the ski base structure is set, while $\bar{R}_{a, y}$ can be considerably higher than $\bar{R}_{a, y s}$ for non-plane ski bases.

In the period between 1995 and 1998 almost 1700 ski base structure measurements were taken of more than 350 skis. The major part of these measurements was performed with the SSA. This unique collection of structure measurements included characterisation of 8 Olympic and 6 World Championship gold medal-winning skis. Results from these measurements will be presented in another paper. 
Table 1. Calculated surface parameters for the measured surface in Figure 6.

\begin{tabular}{|c|c|}
\hline Surface parameter & Value \\
\hline $\begin{array}{l}\text { Characteristic angle } \phi \text { for the measured surface (found by using the bright } \\
\text { point of the 2-D Fourier spectrum of the surface) }\end{array}$ & $\begin{array}{l}-0.171 \\
\text { radians }\end{array}$ \\
\hline $\begin{array}{l}\text { Characteristic period } T \text { for the measured surface (found by using the bright } \\
\text { point of the 2-D Fourier spectrum of the surface) }\end{array}$ & $0.126 \mathrm{~mm}$ \\
\hline $\begin{array}{l}\text { Maximum strength of the bright point in the 2-D Fourier spectrum of the } \\
\text { surface, } \log (|G(u, v)|)\end{array}$ & 8.29 \\
\hline $\begin{array}{l}R_{q} \text { for the whole surface relative to a reference plane parallel with the } \\
x y \text {-plane, } R_{q, 2 D}\end{array}$ & $6.53 \mu \mathrm{m}$ \\
\hline $\begin{array}{l}\text { Mean of the } R_{q} \text { for the columns in the measured surface image, i.e. across } \\
\text { the ski, when each column has an individual reference line parallel to the } \\
y \text {-axis, } \bar{R}_{q, y}\end{array}$ & $5.88 \mu \mathrm{m}$ \\
\hline $\begin{array}{l}\text { Mean of the } R_{q} \text { for the rows in the measured surface image, i.e. along the ski, } \\
\text { when each row has an individual reference line parallel to the } x \text {-axis, } \bar{R}_{q, x}\end{array}$ & $4.78 \mu \mathrm{m}$ \\
\hline $\begin{array}{l}R_{a} \text { for the whole surface relative to a reference plane parallel with the } x y \text { - } \\
\text { plane, } R_{a, 2 D}\end{array}$ & $5.20 \mu \mathrm{m}$ \\
\hline $\begin{array}{l}\text { Mean of the } R_{a} \text { for the columns in the measured surface image, i.e. across } \\
\text { the ski, when each column has an individual reference line parallel to the } \\
y \text {-axis, } \bar{R}_{a, y}\end{array}$ & $4.68 \mu \mathrm{m}$ \\
\hline $\begin{array}{l}\text { Mean of the } R_{a} \text { for the columns in the measured surface image, i.e. across the } \\
\text { ski, when each column has an individual possibly sloping reference line } \\
\text { relative to the } y \text {-axis, } \bar{R}_{a, y s}\end{array}$ & $3.61 \mu \mathrm{m}$ \\
\hline $\begin{array}{l}\text { Mean of the } R_{a} \text { for the rows in the measured surface image, i.e. along the ski, } \\
\text { when each row has an individual reference line parallel to the } x \text {-axis, } \bar{R}_{a, x}\end{array}$ & $3.79 \mu \mathrm{m}$ \\
\hline $\begin{array}{l}\text { Mean of the } R_{a} \text { for the rows in the measured surface image, i.e. along the ski, } \\
\text { when each row has an individual possibly sloping reference line relative to } \\
\text { the } x \text {-axis, } \bar{R}_{a, x s}\end{array}$ & $3.02 \mu \mathrm{m}$ \\
\hline$R_{i}$ for the whole surface, $R_{t, 2 D}$ & $69.00 \mu \mathrm{m}$ \\
\hline $\begin{array}{l}\text { Mean of the } R_{t} \text { for the columns in the measured surface image. i.e. across } \\
\text { the ski, when each column has an individual reference line parallel to the } \\
y \text {-axis, } \bar{R}_{t, y}\end{array}$ & $31.00 \mu \mathrm{m}$ \\
\hline $\begin{array}{l}\text { Mean of the } R_{t} \text { for the rows in the measured surface image, i.e. along the ski, } \\
\text { when each row has an individual reference line parallel to the } x \text {-axis, } \bar{R}_{t, x}\end{array}$ & $25.00 \mu \mathrm{m}$ \\
\hline
\end{tabular}

Many aspects regarding ski base structures were not studied in this paper. Future research will investigate aspects such as the effect of the:

- Structure pressure contact area during skiing

- Structure capillary contact area during skiing under wet snow conditions

- Structure carving area during skiing under hard and dry snow conditions

- Structure bearing area during skiing under soft snow conditions

- Structure pattern on the dilution of water film during skiing under wet snow conditions

- Structure pattern on the minimisation of attachment of dirt during skiing

Furthermore, simulation of the energy dissipation and the contact surface between ski base and snow will be performed.

\section{Conclusions}

A Ski base Structure Analyser (SSA) utilising laser technology has been developed to analyse the detailed structure of a stone-ground ski base. The SSA displays the measured surface as an inage with $739 \times 570$ pixels, where each pixel can have a grey 
level value from 0 to 255 . The value of each pixel is proportional to the height at the corresponding point of the measured surface. Various roughness parameters can be calculated from the measured surface image in order to characterise the surface topography. Some of these parameters are described in this paper. Further the paper contains examples of calculated SSA parameters from a typically measured surface image using MATLAB.

\section{References}

Creath, K. (1988) Phase-measurement interferometry techniques. In Progress in Optics XXVI (E. Wolf, Ed.), Elsevier Science Publishers B.V., s. 349-393.

HAMROCK, B. J. (1994) Fundamentals of fluid film lubrication, McGraw-Hill, New York, 690 p. Mathia, T. G., Midol, A., Lanteri, P. and Longeray, R. (1989) Topography, physicochemistry and wear in sliding of ski soles in regard to rheology of snow. In Proceedings of Eurotrib 89, Elsevier, Amsterdam, pp. 253-260.

MAtHiA, T. G.. ZAHOUANI, H. and MidOL, A. (1992) Topography, wear, and sliding functions of skis. International Journal of Machine Tools Manufacturing, Vol. 32, No. 1/2, pp. 263-266.

Mercer, C. R. and Beheim, G. (1992) Phase-stepping fiber-optic projected fringe system for surface topography measurements. U.S. Patent No. 5,146,293, 6 p.

Moldestad, D.A. (1999) Some Aspects of Ski Base Sliding Friction and Ski Base Structure. Dr. thesis NTNU 1999:137, Norwegian University of Science and Technology, 198 p. 